\title{
The effects of testing methods on the flexural fatigue life of human cortical bone
}

\author{
L.V. Griffin ${ }^{\mathrm{a}, *}$, J.C. Gibeling ${ }^{\mathrm{b}}$, R.B. Martin ${ }^{\mathrm{c}}$, V.A. Gibson ${ }^{\mathrm{c}}$, S.M. Stover ${ }^{\mathrm{d}}$ \\ ${ }^{a}$ Department of Materials Engineering, California Polytechnic State University, San Luis Obispo, CA 93407, U.S.A. \\ ${ }^{\mathrm{b}}$ Department of Chemical Engineering and Materials Science, College of Engineering, University of California, Davis, CA 95616, U.S.A. \\ ' Orthopaedic Research Laboratory, School of Medicine, University of California, Davis, CA 95616, U.S.A. \\ ${ }^{\mathrm{d}}$ Veterinary Orthopedic Research Laboratory, School of Veterinary Medicine, University of California, Davis, CA 95616, U.S.A.
}

\begin{abstract}
A flexural model of four-point bending fatigue that has been experimentally validated for human cortical bone under load control was used to determine how load and displacement control testing affects the fatigue behavior of human cortical bone in three-point and symmetric four-point bending. Under load control, it was predicted that three-point bending produced no significant differences in fatigue life when compared to four-point bending. However, three-point bending produced less stiffness loss with increasing cycles than four-point bending. In four-point bending, displacement control was predicted to produce about one and a half orders of magnitude greater fatigue life when compared to load control. This prediction agrees with experimental observations of equine cannon bone tested in load and displacement control (Gibson et al., 1998). Displacement controlled three-point bending was found to produce approximately a $25 \%$ greater fatigue life when compared to load control. The prediction of longer fatigue life under displacement control may have clinical relevance for the repair of damaged bone. The model can also be adapted to other geometric configurations, including modeling of whole long bones, and with appropriate fatigue data, other cortical bone types.
\end{abstract}

\section{Introduction}

Long bones, such as the femur, experience repetitive loads that involve flexure, torsion, and compression. Additionally, cortical bone is a complex material that is viscoelastic, damage dependent and self-repairing; therefore, the loading history can substantially affect results. Microcracks or related damage, commonly reported in fatigued bone, are a consequence of fatigue loading and are a likely contributor to stress fractures or possibly a mediator for remodeling (Burr et al., 1997; Carter et al., 1977, 1981; Horn, 1993; Pattin et al., 1996, Schaffler et al., 1994a, b; Zioupos et al., 1994). Some of the documented effects of fatigue damage in bone are decreased stiffness, lower strength, and creep relaxation (Carter and Caler, 1985; Martin et al., 1996; Schaffler et al., 1994a, b).
The common configurations for flexural testing of cortical bone are three- and four-point bending. In these experiments, the bone is tested either whole, or machined into small beam samples conforming to ASTM standards for flexural testing. The smaller samples provide a means for determining the material behavior under known loading and serve as a basis for constitutive modeling, whereas the whole bone tests provide information on how the material properties and structure affect performance.

For fatigue testing, the test sample may be repetitively loaded at constant displacement amplitude, known as displacement control, or at constant load amplitude, known as load control. In displacement controlled fatigue, the load is continually being reduced to maintain a constant beam displacement because of fatigue and creep effects. While there are some similarities between these methods, it has been demonstrated for equine cortical bone that displacement controlled loading produces a substantially longer fatigue life in four-point bending 
when compared to load controlled four-point bending (Gibson et al., 1998).

Since there are a variety of flexural test methods, an important question that needs to be addressed is how the testing method affects the results. The answer would be beneficial in the design of experiments as well as in the interpretation of the results, and may provide a means by which to compare the results with those in the literature. A recently developed analytical model was used to investigate the flexural fatigue behavior of cortical bone and has been experimentally validated for fourpoint bending fatigue of cortical bone taken from the human femur in load control (Griffin et al., 1997). The objectives of the current work are to compare fatigue life and damage accumulation characteristics of threeand four-point bending configurations under load and displacement control.

\section{Methods}

The experimental data for the four-point bending under load control were conducted in accordance with ASTM D790 M and a protocol has been developed in our laboratories. The specific details are provided in the literature (Gibson et al., 1995; Griffin et al., 1997; Martin et al., 1996). A complete description of the analytical model is provided elsewhere (Griffin et al., 1997), but a brief description follows. Damage in flexure is assumed to be caused primarily by axial tension and compression stresses and damage caused by shear is neglected. It is further assumed that cortical bone can be modeled as a fiber-matrix composite where the fibers are considered to be complete osteons and the matrix is the interstitial bone composed of primary bone and remodeled osteon fragments. Damage is described in terms which independently account for effects in the fibers and the matrix, and the 'damaged modulus' is obtained using an isostrain rule of mixtures similar to that used in modeling ceramic matrix composites (Ramakrishnan and Jayaraman, 1993)

$E_{\mathrm{bn}}=\left(1-D_{\mathrm{m}}\right) E_{\mathrm{m}} V_{\mathrm{m}}+\left(1-D_{\mathrm{f}}\right) E_{\mathrm{f}} V_{\mathrm{f}}$.

Here, $E_{\mathrm{bn}}$ is the elastic modulus of the bone, $V$ is the volume fraction of osteons (fibers) and $D$ represents damage and the subscripts, $\mathrm{m}$ and $\mathrm{f}$, refer to matrix and osteons (fibers), respectively. $D_{\mathrm{f}}$ and $D_{\mathrm{m}}$ have values that range from zero, associated with an undamaged state, to a maximum value of one, in a failed state.

The matrix damage evolution for tension loading is modeled as a self-limiting process

$\frac{\mathrm{d} D_{\mathrm{m}}}{\mathrm{d} N}=K_{1}\left(D_{\mathrm{s}}-D_{\mathrm{m}}\right)$.

Here, $D_{\mathrm{m}}$ represents the matrix damage, $K_{1}$ is the matrix damage rate parameter, and $D_{\mathrm{s}}=K_{2} \sigma_{\mathrm{a}}^{\mathrm{q}}$ which represents the saturation level of damage to the matrix with $K_{2}$ and $q$ being constants.

Damage to the fibers was assumed to evolve as a positive feedback process that involves the level of damage in the matrix and fiber damage

$\frac{\mathrm{d} D_{\mathrm{f}}}{\mathrm{d} N}=\left(K_{3} D_{\mathrm{f}}+K_{4} D_{\mathrm{m}}\right)$,

where $K_{3}$ is an osteonal damage rate coefficient, and $K_{4}$ is an osteon/matrix damage interaction parameter.

In compression, $D_{\mathrm{c}}$ is used for both damage terms in Eq. (1) and is modeled as

$\frac{\mathrm{d} D_{\mathrm{c}}}{\mathrm{d} N}=K_{5}\left(D_{\mathrm{sr}}+D_{\mathrm{c}}\right)$.

Here, $K_{5}$ is the compression damage rate parameter, $D_{\mathrm{sr}}=K_{6} \sigma_{\mathrm{a}}^{\mathrm{p}}$, which represents incipient damage in the form of stress concentrations, such as Haversian or Volkmann canals, which serve to initiate, or extend $D_{\mathrm{c}}$, and $p$ is the exponent of the stress dependence.

The elastic modulus (Eq. (1)) as a function of stress $(\sigma)$ and the number of cycles $(N)$ requires experimental data to determine the parameters, $K_{i}, p$, and $q$ applicable for cortical bone. These are determined by substituting Eqs. (2)-(4) into (1) and performing a nonlinear regression of the resulting equation to fatigue data. The parameters for the present analysis were derived from the human femur data of Pattin et al. (1996), and the details are presented elsewhere (Griffin et al., 1997).

The development of the flexural fatigue model was based on laminated beam theory (Agarwal and Broutman, 1990). In this case, the lamellae represent regions of different levels of damage, hence different elastic moduli, rather than regions of different initial material properties. By use of a discretized laminated beam, the modulus can vary throughout the beam as a function of the number of cycles $(N)$, stress $(\sigma)$, and spatial position $(x$ and $y$ ). Assuming that the beam is simply supported, the differential equation for the beam deflection, $v$, can be shown to be

$\frac{\mathrm{d}^{2} v}{\mathrm{~d} x^{2}}=\frac{M}{\gamma-\left(\beta^{2} / \alpha\right)}$,

where $\alpha, \beta$, and $\gamma$ are functions of the elastic properties and geometry of the section as described elsewhere (Griffin et al., 1997).

The beam model is numerically integrated to find the deflection using a previously developed technique (Mischke, 1978). For four-point bending with symmetrically placed supports and a center span of $L / 2$, the total applied load for the beam as calculated using beam theory is

$P=\frac{2 E_{\mathrm{bn}} b h^{2} \varepsilon_{\mathrm{max}}}{3 L}$. 
Here $\max _{\text {ax }}$ is the desired peak flexural strain, and $b, h$ and $L$ are the width, depth, and supported length of the beam, respectively. For three-point bending, assuming symmetric loading, the applied load to the beam is

$P=\frac{4 E_{\mathrm{bn}} b h^{2} \max }{3 L}$.

For both three- and four-point bending, only half the beam needs to be considered due to the symmetry of loading. For load control, the procedure involves first calculating the flexural rigidity $\left(\gamma-\beta^{2} / \alpha\right.$ in Eq. (5)) for each segment of the beam. The elastic modulus of each segment is calculated every cycle by using Eqs. (1)-(4) depending on whether the loading was tension or compression. After calculating the flexural rigidity, Eq. (5) is numerically integrated to find the deflection.

For displacement control, the procedure is similar to that of load control except that the load is not known from one cycle to the next because the increase of damage reduces the stiffness of the beam, and consequently the load. Therefore, an extra iterative step that involves incremental application of the load is needed until the desired displacement limit is obtained. After the load is calculated for the cycle, the procedure is simply like that of load control: the damage is assessed, and if beam failure has not occurred, the process is repeated until failure is reached.

\section{Results}

The fatigue life predictions for three- and four-point bending in load control are nearly identical. Fig. 1 compares the fatigue life behavior of three- and four-point bending in displacement control for initial flexural strains ranging from 5500 to $7500 \mu \varepsilon$. It is interesting to note that there is little difference between the stiffness loss for both beams until the last stage of failure, where the modulus drops rapidly. In the case of load control, this final failure event is longer for displacement control than load control. In Fig. 2a and b, only one half of the beam is shown since the loading is symmetric. The damage is gray-scale coded with black indicating complete failure. For three-point bending, the damage is much more localized than four-point bending that spans much of the length. In Fig. 3a, the model predictions for human femur under displacement controlled four-point bending at 7500 and $5500 \mu \varepsilon$ graphically show the longer fatigue life when compared to load control. Shown in Fig. 3b are experimental data for equine metacarpal bone tissue that was loaded in displacement control and load control at $10000 \mu \varepsilon$. The model predictions for human cortical bone are qualitatively consistent with experimental observations for equine metacarpal bone. Both show a very much longer fatigue life for displacement control loading,

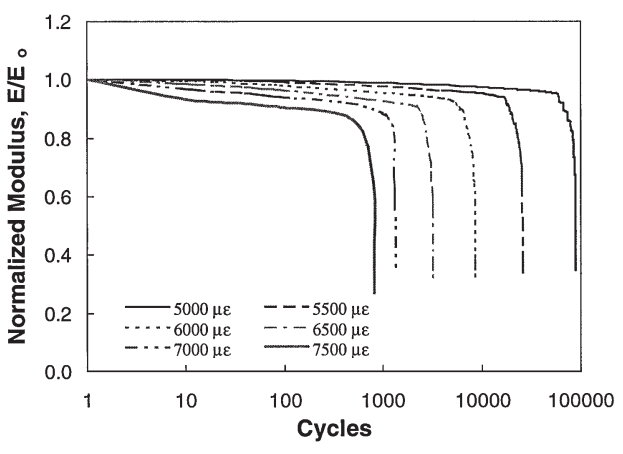

(a)

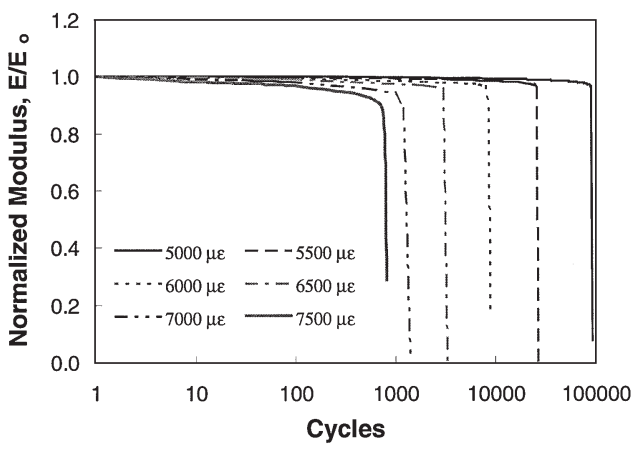

(b)

Fig. 1. (a) Load control fatigue life predictions for four-point bending and (b) Load control fatigue life predictions for three-point bending.

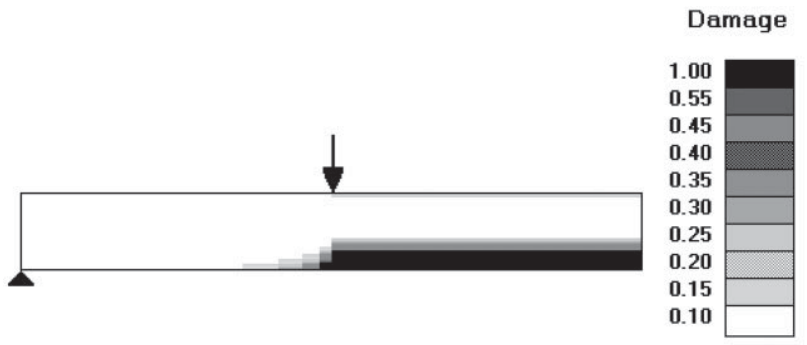

(a)

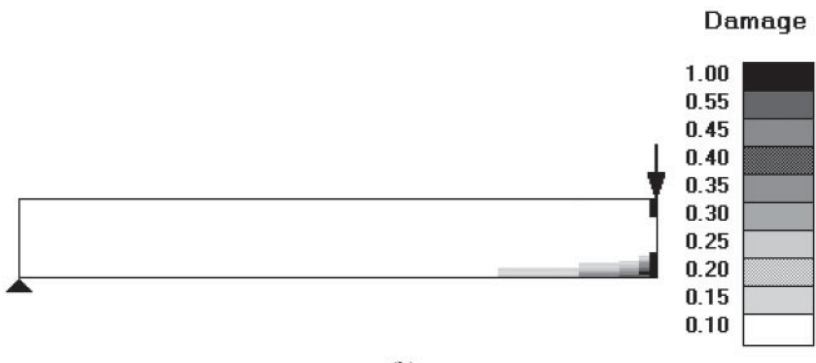

(b)

Fig. 2. (a) Predicted damage zone for at $N=N_{\mathrm{f}}-1$ for four-point bending and (b) Predicted damage zone for three-point bending at $N=N_{\mathrm{f}}-1$.

slightly less initial stiffness loss for displacement control, and similar damage accumulation rates for displacement control in the final stage of failure. 


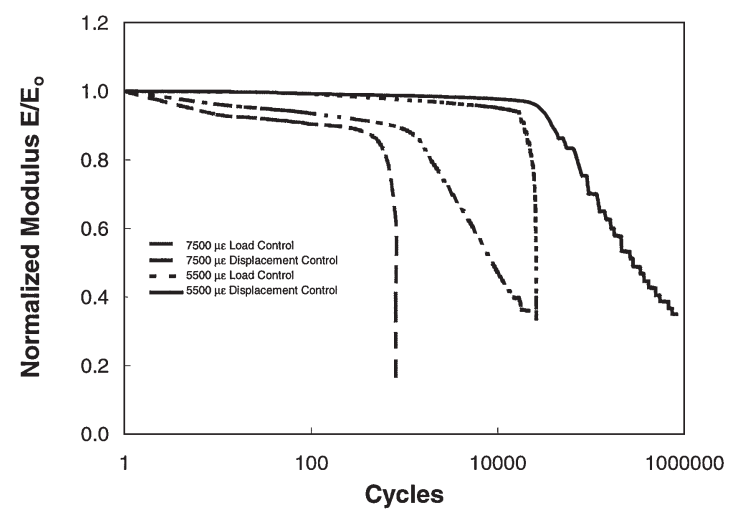

(a)

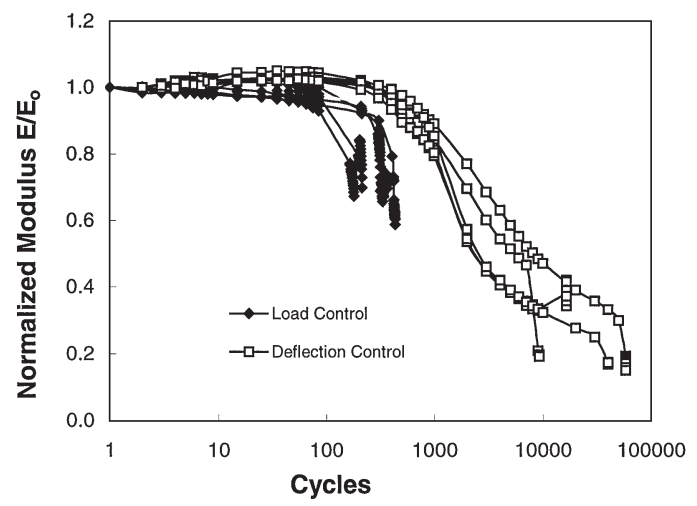

(b)

Fig. 3. (a) Model predictions for human femoral bone under four-point bending in load and displacement control at 5500 and $7500 \mu \varepsilon$. (b) Experimental data for equine cannon bone in load and displacement control at $10000 \mu \varepsilon$ tested under four-point bending (Gibson et al., 1997).

\section{Discussion}

The objectives of this research were to use an analytical model to determine how flexural test configuration and loading method would influence the predicted fatigue life of cortical bone. The model shows that the damage is not uniform and is strongly dependent on the loading configuration (Fig. 2). To compare the model predictions to experimental data, a damage modulus based on the beam deflection is calculated using beam theory assuming that the beam elastic modulus is uniform. This damaged modulus is normalized with the initial elastic modulus $\left(E / E_{\mathrm{o}}\right)$ which then allows for comparisons of model predictions to experiments that use the same assumption.

In four-point bending, there is a large region of damaged bone between the inner supports that is a result of that material being exposed to the maximum flexural stresses. This large region of damaged bone produces a substantial increase in apparent beam compliance during the fatigue test. In three-point bending, the maximum flexural stress is at the point of load application, but linearly decreases to zero at the outer supports. The result is a relatively small region of damaged bone, which does not greatly affect the stiffness of the beam.

The model fatigue life predictions for three-point bending in displacement control were somewhat unexpected. While displacement control was shown to produce a longer fatigue life for three-point bending (on the order of $25 \%$ ) when compared to load control, this difference is not nearly as profound as the one to two order of magnitude greater fatigue life seen in fourpoint bending. The explanation for this behavior can be found by considering how damage accumulates in threepoint bending as compared to four-point bending (Fig. 2). Since damage in three-point bending is concentrated near the point of load application, the overall beam stiffness is not greatly affected and the load is not substantially reduced with increasing number of cycles, as in four-point bending, resulting in prolonged high stresses that lead to a more rapid failure in the former case.

In displacement control mode, the load is reduced continually throughout the fatigue life because as damage accumulates the beam becomes more compliant. While we have no human femur data for load control four point bending, a recent investigation for equine cannon bone has demonstrated that the fatigue life in displacement controlled four-point bending is as much as two orders of magnitude longer when compared to load control. This qualitative agreement is shown in Fig. 3. Also, Carter and coworkers $(1981,1983)$ and Pattin et al. (1996) have shown that strain control produces a longer fatigue life than stress control which agrees qualitatively with our predictions.

A possible weakness of the current formulation may be associated with the fact that damage due to shear load was neglected. One of the fundamental assumptions was that damage was due to axially applied loads in tension or compression and not shear. Shear was neglected for two reasons: (1) we wanted to see if the primary effects of fatigue damage in bending were dominated by tension and compression, and (2) we had no data on damage accumulation in shear. In four-point bending, this is probably a reasonable assumption since the shear stress due to direct shear is zero between the inner supports. For the configuration under consideration, that is half the beam length. In threepoint bending, neglecting shear stress may be a poor assumption. However, if damage due to shear stress produces a strong effect, it is reasonable to assume that the actual fatigue life would be no greater than that predicted by the model.

Another factor that may contribute to a shortened fatigue life in three-point bending is that there is a very complicated stress state at the point of contact. Even if shear stress is not important, three-point bending may still produce a shorter fatigue life than predicted using 
this model because of contact stresses. From Eqs. (6) and (7), it can be seen that for three-point bending, the load which will produce an equivalent flexural strain at the point of load contact is four times that of four-point bending, since the overall load in four-point bending, $P$, is divided equally between the two load points. These factors suggest that four-point bending is a more desirable testing configuration for bone as it avoids an unphysiologic stress concentration.

When conducting tests for material behavior, it is important to understand how the testing method influences the data. Furthermore, bone is a very complicated material that exhibits a variety of documented nonlinear effects such as viscoelasticity, creep, and damage-induced stiffness reduction (Carter and Caler, 1981; Fondrk et al., 1988; Gibson et al., 1996; Lakes and Katz, 1979; Pattin et al., 1996). Because we were evaluating our damage formulation, some of these effects were not included in this study, although the model could accommodate these. However, to obtain the cycle damage, the load would need to be applied incrementally, and the damage at each point would need to be determined by iteration, in a manner similar to that of the finite element method.

Some of the useful features of this fatigue model are the ability to handle specific bending geometry and flexibility to be adapted to various materials. All that would be required is that beam theory should be a reasonable approximation. The model could easily be adapted to model the fatigue of a long bone such as the human femur, tibia, or horse metacarpus. To be adapted to other bone types, fatigue data for uniaxial stress control data such as that of Pattin and coworkers are needed (1996).

Our model predicts that load controlled tests in either three- or four-point bending produce similar fatigue lives, but stiffness loss in three-point bending is less than that of four-point bending. The fatigue life in three-point bending is slightly longer under displacement control than load control. In four-point bending, displacement control can produce a fatigue life up to two orders of magnitude greater than load control at the same initial flexural strain.

\section{Acknowledgements}

This work was supported by NIH grant AR41644. We also acknowledge the assistance of the Hearst Foundation, Mr. and Mrs. Amory J. Cooke, the California Horse Racing Board Postmortem program, and the California Center for Equine Health and Performance, University of California, Davis, with funds provided by the Oak Tree Racing Association, the State of California satellite wagering fund, and contributions by private donors.

\section{References}

Agarwal, B.D., Broutman, L.J., 1990. Analysis and Performance of Fiber Composites, 2nd ed. Wiley, New York.

Burr, D.B, Forwood, M.R., Fyhrie, D.P., Martin, R.B., Schaffler, M.B., Turner, C.H., 1997. Bone microdamage and skeletal fragility in osteoporotic and stress fractures. Journal of Bone Mineral Research $12,6-15$.

Carter, D.R., Hayes, W.C., 1977. Compact bone fatigue damage: a microscopic examination. Clinical Orthopedic Related Research 127, 265-274.

Carter, D.R., Caler, W.E., 1983. Cycle-dependent and time-dependent bone fracture with repeated loading. Journal of Biomechanical Engineering 105, 166.

Carter, D.R., Caler, W.E., Spengler, D.M., Frankel, V.H., 1981. Fatigue behavior of adult cortical bone: The influence of mean strain and strain range. Acta Orthopedica Scandinavica 52, 481-490

Choi, K., Goldstein, S.A., 1992. A comparison of the fatigue behavior of human trabecular and cortical bone tissue. Journal of Biomechanics $25,1371-1381$

Fondrk, M., Bahniuk, E., Davy, D.T., Michaels, C., 1988. Some viscoplastic characteristics of bovine and human cortical bone. Journal of Biomechanics 21, 623-630.

Gibson, V.A., Stover, S.M., Martin, R.B., Gibeling, J.C., Willits, N.H., Gustafson, M.B., Griffin, L.V., 1995. Fatigue behavior of the equine third metacarpus: mechanical property analysis. Journal of Orthopedic Research 13, 861-868.

Gibson, V.A., Stover, S.M., Martin, R.B., Gibeling, J.C., Griffin, L.V., 1996. Fatigue behavior of the equine third metacarpal bone tissue: load-control vs deflection-control. Trans ORS 21, 611.

Griffin, L.V., Gibeling, J.C., Martin, R.B., Gibson, V.A., Stover, S.M., 1997. A model of flexural fatigue damage for cortical bone. Journal of Orthopaedic Research 15, 604-615

Horn, T.S., 1993. The identification of cortical microdamage in fatigueloaded bone using a non-invasive impulse response vibration testing technique. Bone 14, 259-264.

Lakes, R.S., Katz, J.L., 1979. Viscoelastic properties of wet cortical bone: Part II, relaxation mechanisms. Journal of Biomechanics 12, 679-687.

Martin, R.B., Stover, S.M., Gibson, V.A., Gibeling, J.C., Griffin, L.V., 1996. In vitro fatigue behavior of the equine third: remodeling and microcrack damage analysis. Journal of Orthopaedic Research 14, 794-801.

Mischke, C.R., 1978. An exact numerical method for determining the bending deflection and slope of stepped shafts. In: Advances in Reliability and Stress Analysis. Proc Wtr Mtg ASME, San Fran, pp. 101-115.

Pattin, C.A., Caler, W.E., Carter, D.R., 1996. Cyclic mechanical property degradation during fatigue loading of cortical bone. Journal of Biomechanics 29, 69-79.

Ramakrishnan, V., Jayaraman, N., 1993. Mechanistically based fatiguedamage evolution model for brittle matrix fibre-reinforced composites. Journal of Material Science 28, 5592-5602.

Schaffler, M.B., Pitchford, W.C., Choi, K., Riddle, J.M., 1994a. Examination of compact bone microdamage using back-scattered electron microscopy. Bone 15, 483-488.

Schaffler, M.B., Choi, K., Milgrom C., 1994b. Microcracks and aging in human femoral compact bone. In Transactions of the 40th Annual Meeting of the Orthopaedic Research Society, vol. 19, pp. 190.

Stover, S.M., Gibson, V.A., Martin, R.B., Gibeling, J.C., Griffin, L.V., 1995. Osteonal pullout is associated with fatigue resistance of equine third metacarpal bone tissue. Trans ORS 20, 129.

Zioupos, P., Currey, J.D., Sedman, A.J., 1994. An examination of the micromechanics of failure of bone and antler by acoustic emission tests and laser scanning confocal microscopy. Medicine Engineering and Physics 16, 203-212. 